\title{
Glucose transporter expression and the potential role of fructose in renal cell carcinoma: A correlation with pathological parameters
}

\author{
LUIS M. ANTÓN APARICIO ${ }^{1,2}$, VANESSA MEDINA VILLAAMIL ${ }^{3}$, MOISÉS BLANCO CALVO ${ }^{3}$, \\ LUIS VALBUENA RUBIRA ${ }^{4}$, JOSÉ MANUEL ROIS, MANUEL VALLADARES-AYERBES ${ }^{1}$, \\ ROSARIO GARCÍA CAMPELO ${ }^{1}$, M. VICTORIA BOLÓS ${ }^{5}$ and ENRIQUE GRANDE PULIDO ${ }^{6}$ \\ ${ }^{1}$ Medical Oncology Department, A Coruña University Hospital; ${ }^{2}$ Department of Medicine, University of A Coruña;
${ }^{3}$ Biomedical Research Institute (INIBIC), A Coruña; ${ }^{4}$ Pathology Department, Modelo Hospital, A Coruña;
${ }^{5}$ Pfizer Oncology, Madrid; ${ }^{6}$ Medical Oncology Department, Ramón y Cajal Hospital, Madrid, Spain
}

Received December 24, 2009; Accepted May 20, 2010

DOI: $10.3892 / \mathrm{mmr}_{0} 00000300$

\begin{abstract}
All mammalian cells contain one or more members of the facilitative glucose transporter (GLUT) gene family. Glucose transporter membrane proteins (GLUT) regulate the movement of glucose between the extracellular and intracellular compartments, maintaining a constant supply of glucose available for metabolism. Tumor cells are highly energy-dependent, therefore GLUT overexpression is often observed. In fact, overexpression of GLUT1 has been correlated with hypoxia markers in several tumor types, including renal cell carcinoma (RCC). We retrospectively analyzed 80 paraffin-embedded RCC samples. The pattern of GLUT1-5 expression in RCC specimens was evaluated using tissuearray technology and correlated with histological tumor characteristics. Pathological parameters included tumor location, renal pelvis, vein and lymph vessel invasion, capsule breakage, histological subtype, Furhman grade, hilar invasion and tumor stage at diagnosis. The expression of five facilitative glucose transporters, GLUT1 (erythrocyte type), GLUT2 (liver type), GLUT3 (brain type), GLUT4 (muscle/fat type) and GLUT5 (small intestinal type), was semi-quantitatively analyzed. In non-parametric, Mann-Whitney U and KruskalWallis tests, a significant positive correlation was consistently found between moderately differentiated RCC tissues and the expression of GLUT5 ( $\mathrm{p}=0.024)$. Patients who had pelvic invasion and capsule breakage at diagnosis also showed increased GLUT5 expression levels $(\mathrm{p}=0.039$ and $\mathrm{p}=0.019$, respectively). Moreover, GLUT5 showed statistical significance in those samples identified as being of clear cell histological
\end{abstract}

Correspondence to: Dr Luis M. Antón Aparicio, A Coruña University Hospital, As Xubias 84, 15006 A Coruña, Spain

E-mail: luis.m.anton.aparicio@sergas.es

Key words: carbohydrates, glucose transporter, renal cell carcinoma, tissue-arrays type ( $\mathrm{p}=0.001)$. A high expression of GLUT5 in human RCC was observed. GLUT5 appears to be correlated with grade II differentiation, locoregional invasion and aggressiveness, and may play a role in RCC development.

\section{Introduction}

The worldwide incidence of renal cell carcinoma (RCC) has increased in the past three decades (1). Its frequency is higher in men than in women (2). It is estimated that 63,300 and 54,390 patients are diagnosed every year in the EU and US, respectively, of which 39,000 eventually succumb to the disease $(3,4)$.

Glucose metabolism is a central component of living systems; the oxidation of glucose represents a major source of metabolic energy for mammalian cells. However, tumor cells have a reduced ability to use oxidative metabolism and rely instead on an increased rate of glycolysis and glucose use (5). A more active glycolytic metabolism is reflected in an increased rate of glucose uptake (6). This differential glucose uptake between normal and tumor cells is the basis for the diagnosis of various tumors by positron emission tomography using the glucose analogue fluorine-18-fluorodeoxyglucose (7).

Since plasma membrane is impermeable to polar molecules such as glucose, glucose uptake necessitates the use of membrane-associated carrier proteins.

The glucose transporter (GLUT) protein family consists of 14 members grouped into three major classes based on sequence homology and substrate selectivity (8). GLUT proteins mediate the transport of glucose and other monosaccharides into and out of mammalian cells according to a concentration gradient (9). GLUT transporters have different expression patterns in different tissues and play various roles according to substrate specificities, kinetic properties, function and developmental regulation. The expression of five facilitative glucose transporter genes, GLUT1 (erythrocyte type), GLUT2 (liver type), GLUT3 (brain type), GLUT4 (muscle/ fat type) and GLUT5 (small intestine type), is relatively tissue specific. Certain tissues and cells express two or even more 
glucose transporter isoforms, indicating that the organism has developed a backup system guaranteeing adequate energy supply (10). Several members of the GLUT family transport not only glucose, but also fructose (GLUT2, -5, -7, -8, -9, -11 and -12), except GLUT5, which exclusively transports fructose.

It is currently accepted that the increase in glucose uptake observed in malignant cells is associated with the overexpression of GLUTs. Oncogenes and growth factors are responsible for GLUT overexpression in malignant cells (8). Increase in glucose transport and metabolism may reflect an adaptative requirement by these rapidly growing cells for more sources of energy (11). Glucose provides much-needed energy for the cells in the following ways: it serves as a primary substrate for the generation of ATP, supplies a carbon source for the synthesis of other macronutrients, such as nucleic acids and phospholipids, and is metabolized by the pentose phosphate pathway to generate NADPH.

GLUTs transport glucose, and increased expression of these transporters has been reported in various tumor types. GLUT1 has been found overexpressed in breast, colorectal, lung, and ovarian carcinoma (12), GLUT12 in breast cancer (13) and GLUT3 in lung, ovarian and gastric cancers (14).

Less is known regarding the role of the GLUT system in RCC development. Previous studies (15) compared normal kidney and RCC tissue samples in terms of expression levels of GLUT members measured by reverse transcriptasepolymerase chain reaction (RT-PCR). While kidney normal tissue expressed all the GLUT isoforms in the medium range, it was shown that, in clear cell RCC, the expression of GLUT1 was increased significantly. However, the levels of expression of GLUT4, 9 and 12 were found to be decreased. GLUT4 was also found to be elevated in chromophobe histological subtypes. A study of 187 tumor tissue samples of kidney cancer (16) also found that GLUT1 expression, measured by immunohistochemistry in a panel of tissue microarrays, was correlated with a reduced overall survival and higher levels of hypoxia-inducible factor-1 (HIF-1 $\alpha$ ).

However, these findings have uncertain clinical significance, and could not be correlated in a prospective manner when fluorine-18-fluorodeoxyglucose-positron emission tomography (FDG-PET) was used as a diagnostic technique in 19 patients before nephrectomy. Only 6 out of the 19 histologically confirmed patients had a positive FDG-PET result, therefore the clinical and biological significance of GLUT1 expression in kidney cancer has yet to be determined (17). The aim of the present study was to determine the immunohistochemical pattern of expression of the glucose transporters 1, 2, 3, 4 and 5 (GLUT1-5) using a tissue array (TA) in tumor tissue samples with different RCC histological subtypes, and to evaluate whether GLUT1-5 expression correlates with different histopathological parameters: tumor location, renal pelvis, vein and lymph vessel invasion, capsule breakage, histological subtype, Furhman grade, hilar invasion and tumor stage.

\section{Materials and methods}

Patients and tumor samples. The clinical and pathological data of patients who were diagnosed with RCC and underwent surgery at the Department of Urology of Modelo Hospital, A Coruña, Spain, from 1996 to 2007, were reviewed. The study group consisted of 80 patients whose original pathological specimens were available for evaluation. The Institutional Review Board of Modelo Hospital approved the retrospective review of the medical records and the use of archived tumor specimens.

Tissue microarray generation. All archival tissue samples were routinely fixed in formalin and embedded in paraffin. Representative tissue areas were marked on standard $\mathrm{H} \& \mathrm{E}$ sections, punched out of the paraffin block using a $2.0-\mathrm{mm}$ punch and inserted in a recipient paraffin block to produce a $6 \times 8$ array of 48 cases. A normal cerebellum tissue sample was inserted as a negative control. When possible, triplicate cores per specimen were arrayed on a recipient paraffin block in order to decrease the error introduced by sampling and to minimize the impact of tissue during processing. Sections (4 $\mu \mathrm{m})$ were cut from the completed array blocks and transferred to silanized glass slides.

Immunohistochemistry. The working dilution was determined using positive controls, as indicated in the literature. For GLUT1, esophagus was used; for GLUT2, liver was used; for GLUT3, placenta was used, for GLUT4, heart was used and for GLUT5, small intestine was used. Additional sections, run in parallel, but with the omission of the primary antibody, served as negative controls.

The tissue sections were deparaffinized by incubation in xylene and rehydrated in a graded series of ethanol and water solutions. The antigen was retrieved with $0.01 \mathrm{M}$ citrate buffer ( $\mathrm{pH}$ 6.0) by heating the samples in a microwave vacuum histoprocessor (2100 Retriever ${ }^{\mathrm{TM}}$; PickCell Laboratories) at a controlled final temperature of $121^{\circ} \mathrm{C}$ for $15 \mathrm{~min}$. The primary antibodies were diluted in Dako antibody diluent (Dakocytomation) with background-reducing components and were used at the followings dilutions: GLUT1-2, 1:50, GLUT3, 1:25 and GLUT4-5, 1:250 (all Abcam). The primary antibodies were incubated at room temperature for $30 \mathrm{~min}$ and detected using the Dako EnVision system and diaminobenzidine according to the manufacturer's instructions.

Semi-quantification of antibody staining. The immunoreactivity score was evaluated, following other groups, by multiplying the percentage of positive cells (PP percentage) and the staining intensity (SI). First, the PP percentage was scored as: $0,<1 \% ; 1,1-24 \% ; 2,25-49 \% ; 3,50-74 \%$; and 4 , $\geq 75 \%$. Second, the SI was scored as: 1 , weak; 2 , medium; and 3 , intense staining.

Each slide was carefully examined in the area of the tumor that contained the greatest fraction of positively stained cancer cells.

Statistical analysis. Data are expressed as the mean \pm standard deviation (SD). The statistical significance of the differences found was evaluated at the $95 \%$ confidence level by nonparametric statistical, Mann-Whitney $U$ and Kruskal-Wallis tests. p-values of $<0.05$ were considered the cut-off point for significance. All statistical analyses were performed using commercially available software (SPSS 17.0 for Windows). 
Table I. Clinical characteristics of the patients.

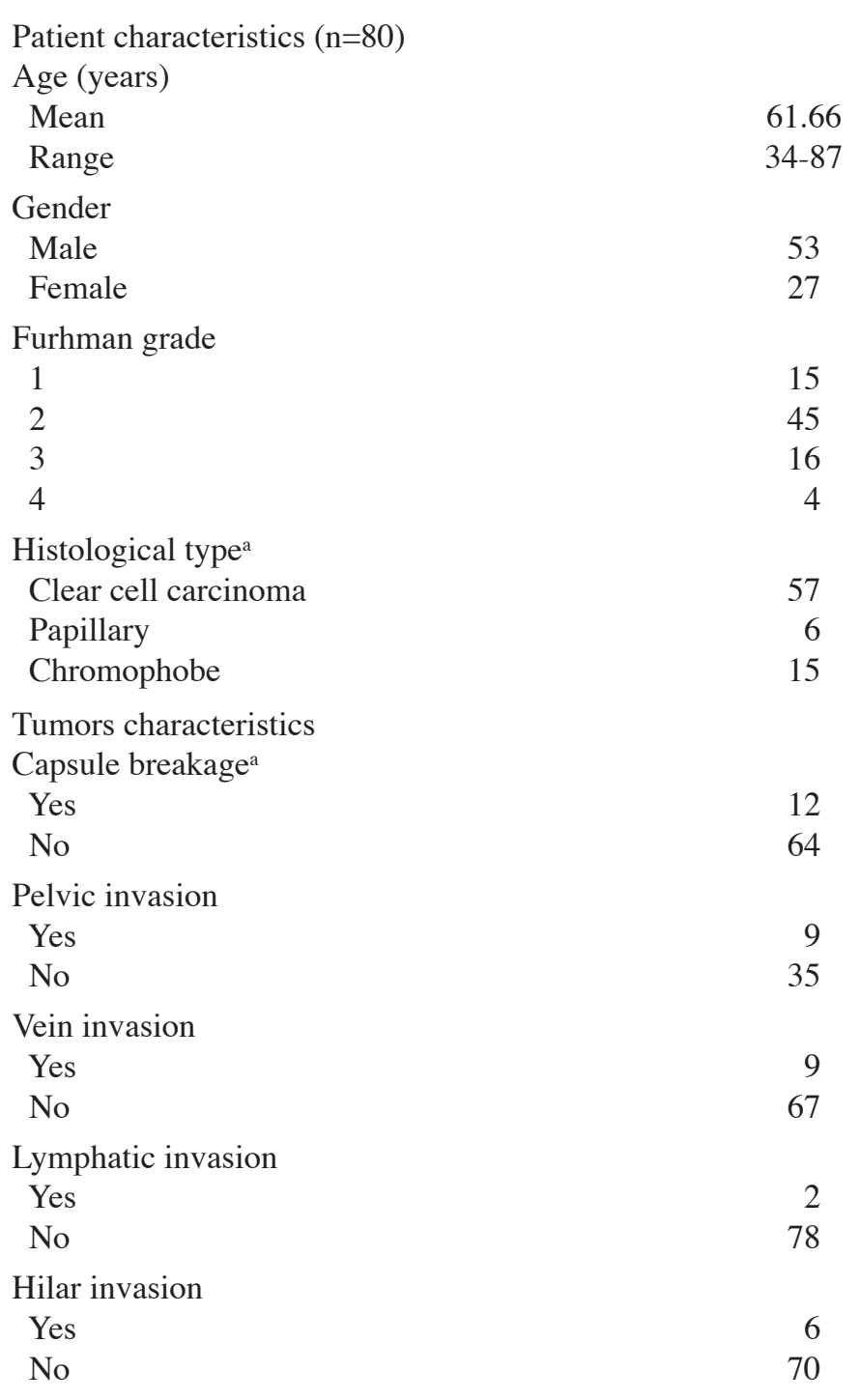

aTwo samples had an unknown histologic type. Capsule breakage, vein and hilar invasion could not be determined in four samples.

\section{Results}

The clinical characteristics of the patients are detailed in Table I. The expression of GLUT isoforms was analyzed by immunohistochemistry in the tumor tissue samples (Figs. 1-3).

GLUT1 showed membranous staining in red blood cells (Fig. 1A). Positive but weaker staining was also observed in 38 of $80(47.5 \%)$ RCC samples (Fig. 1A), in which GLUT1 staining was observed in the plasmatic membrane and cytoplasm. Tubular epithelium, glomerulus and interstice were negative for GLUT1 staining. There was no correlation between GLUT1 staining and the pathological parameters considered for review such as grade of differentiation, pelvic invasion and capsule breakage (Table II).

GLUT2 was detected as a strong reaction in the cell membrane in 58/80 (72.5\%) RCC samples. A cytoplasmic reaction was observed with moderate intensity in $27.5 \%$ of the RCC samples (Fig. 1B). No significant correlation between the histological parameters studied and the expression of GLUT2 was observed (Table II).

GLUT3 was detected in 30/80 (37.6\%) RCC samples, showing a weak cell membrane reaction and granular cytoplasmic staining (Fig. 2A). No significant correlation between the histological parameters studied and the expression of GLUT2 or GLUT3 was observed (Table II).

GLUT4 was detected in a weak cytoplasmic pattern in 45/80 (56.3\%) RCC samples (Fig. 2B). Patients classified as T4 showed increased GLUT4 expression $(\mathrm{p}=0.029)$.

By contrast, GLUT5 showed high intensity in the membrane and cytoplasm of tumoral cells in 46/80 (57.6\%) RCC samples (Fig. 3B and C). In normal kidney, the intensity reaction on the apical pole of tubular epithelium (Fig. 3A), vascular endothelium, glomerulus and interstice was negative. A significant positive correlation was found between moderately differentiated RCC tumor tissues and GLUT5 ( $\mathrm{p}=0.024)$. Patients with pelvic invasion showed increased GLUT5 expression $(\mathrm{p}=0.039)$. A significant positive correlation was observed between GLUT5 expression and patients with capsule breakage $(\mathrm{p}=0.019)$. Related to histological type, GLUT5 was found to present higher expression in clear RCC ( $\mathrm{p}=0.001)$ (Table II).

\section{Discussion}

The study of GLUT1-5 expression in RCC using TA was viable and successful (16). Tumoral heterogeneity is partly obviated when evaluating different factors using TA.

This study investigated the expression and potential roles of five family glucose transporters (GLUT1-5) in RCC. Facilitative GLUTs transport of glucose and increased expression of these transporters has been reported in RCC and other tumor types.

Our results provide evidence that human RCC exhibits different levels and patterns of expression of GLUT isoforms. Moreover, we found that a substantial proportion of the tumor specimens expressed increased levels of GLUT5.

Although pre-clinical approaches to explore the role of GLUT5 in RCC are required, the significant correlation of immunohistochemical GLUT5 expression in human tumor samples with RCC tumor differentiation grade, pelvic invasion and capsule breakage (Table II) suggests that fructose uptake mediated by GLUT5 may play a role in the progression of RCC.

A previous study (15) using molecular methods demonstrated that GLUT5 is overexpressed in clear cell RCC, although not significantly. However, we found significantly higher levels of GLUT5 in clear cell RCC compared to chromophobe and papillary histological types.

GLUT5 is the best characterized fructose transporter and is expressed in various cell types and tissues (intestine, testis, kidney, skeletal muscle, fat and brain) (11). Among the seven members capable of transporting fructose, GLUT5 is the sole transporter specific for fructose with no ability to transport glucose or galactose. Currently, interest in GLUT5 is increasing due to a dramatic increase in the total fructose consumption in the human diet (10).

Fructose is the sweetest of all natural sugars. It is absorbed in the jejunum and transferred through the apical membrane of epithelial cells into the portal circulation. In the liver, fructose 
A

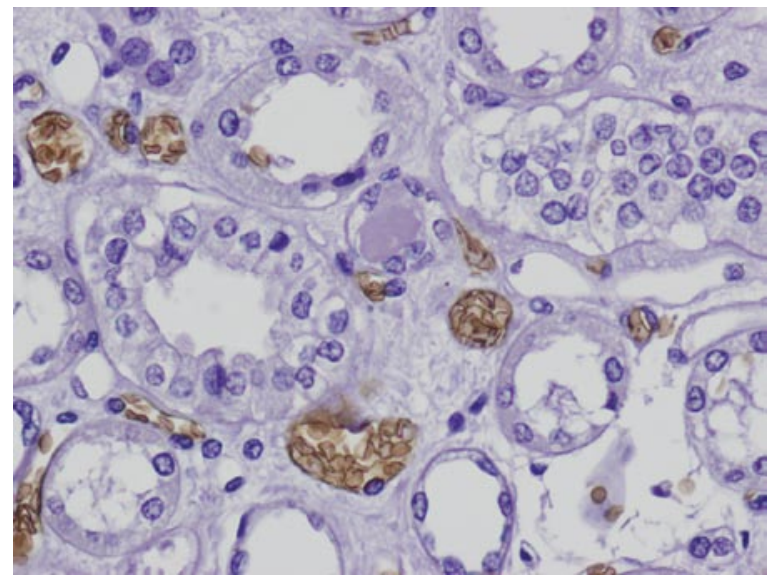

B

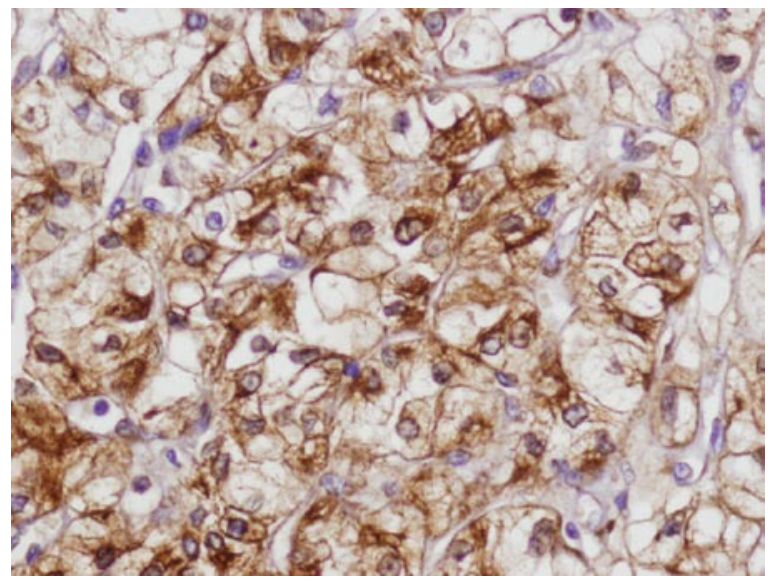

Figure 1. (A) GLUT1 immunostaining in RCC. The tubular epithelium, glomerulus and interstices were negative (x40), while the intravascular erythrocytes were positive. (B) GLUT2 immunostaining in RCC. Moderate cytoplasmic positivity was observed in $40 \%$ of tumor cells (x50).

A

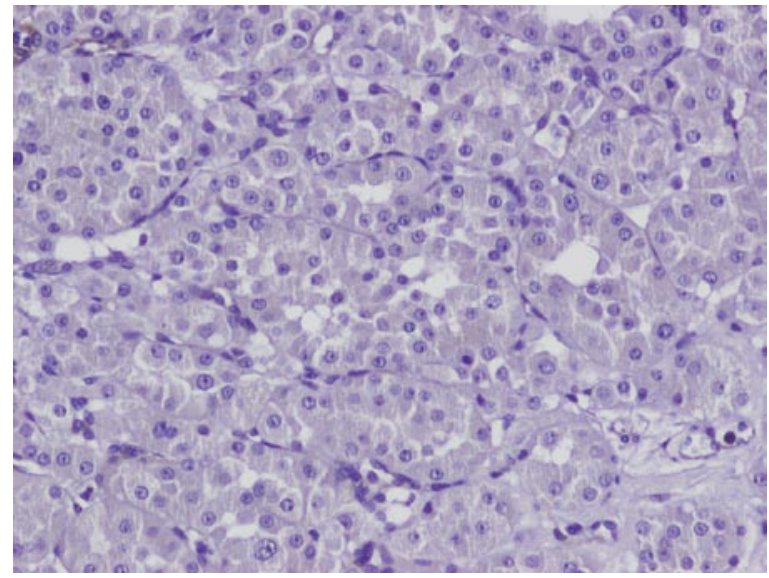

B

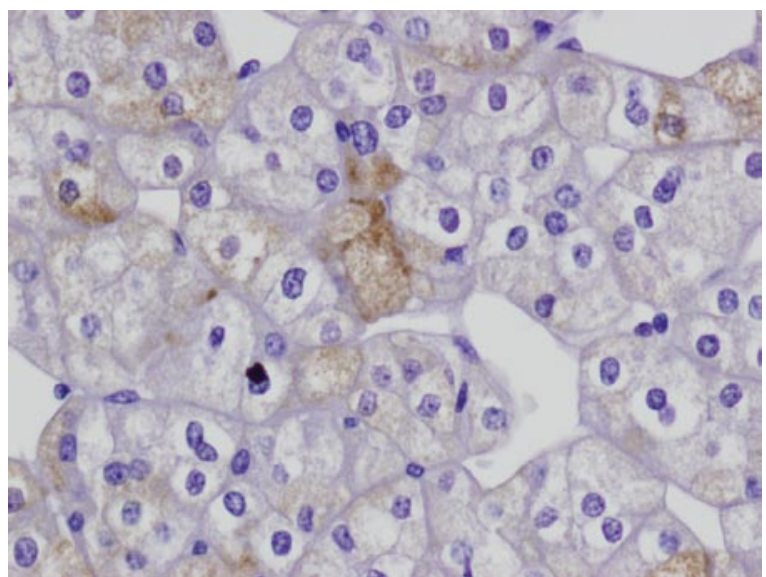

Figure 2. (A) Negative immunostaining for GLUT3 in granular cell renal carcinoma (x40). (B) GLUT4 immunostaining in RCC. Light cytoplasmic positivity was observed in $10 \%$ of tumor cells.

A

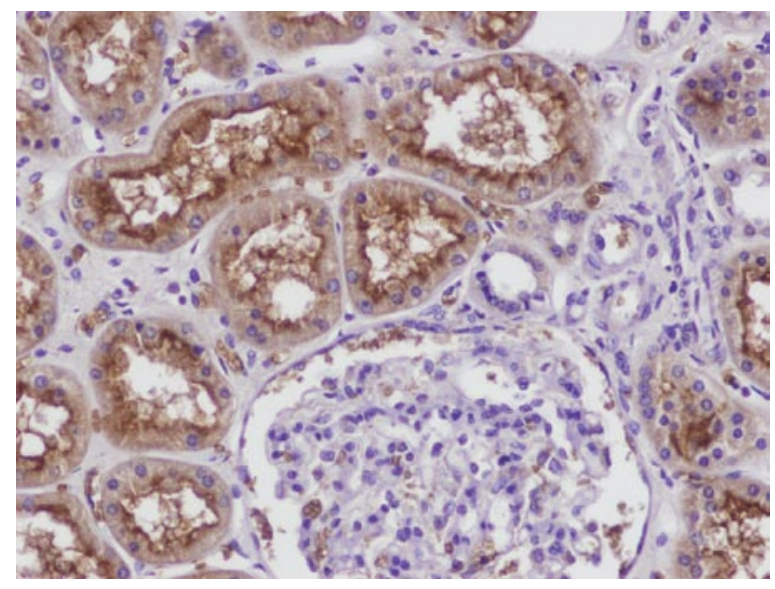

B

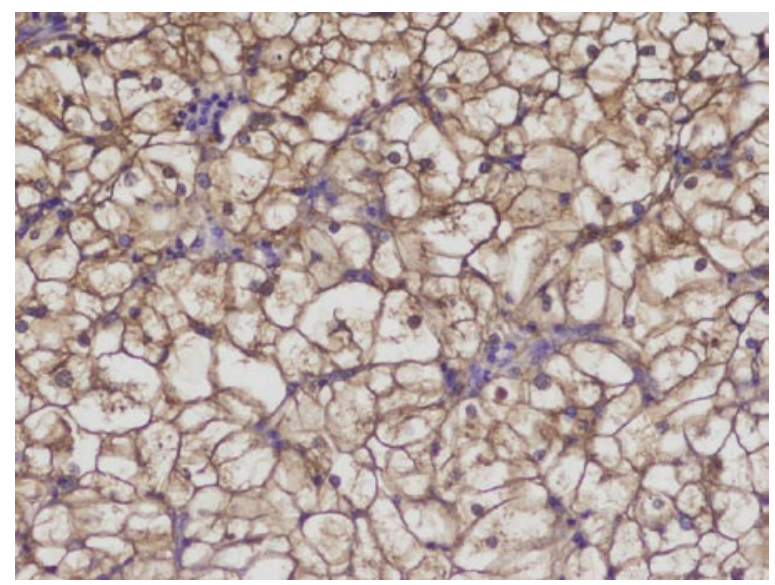

Figure 3. GLUT5 immunostaining. (A) Normal kidney with intense positivity in the apical pole of tubular epithelium. The glomerulus, the interstices and the vascular endothelium were negative (x40). (B) Intense membrane positivity was observed in most tumor cells (x50). (C) Intense cytoplasmic positivity was observed in most tumor cells (x50).

is phosphorylated to fructose 1-phosphate and converted to glycerol 3-phosphate for the synthesis of glycerol, or metabolized to acetyl-CoA and incorpored into fatty acids. Only a small portion is converted into glucose.
After the small intestine, the kidney expresses the majority of GLUT5 protein in humans. GLUT5 is present in the apical plasma membrane of S3 proximal tubule cells (18), where it has the potential to recapture fructose lost from glomerular 
Table II. Relationship between GLUT1-5 expression and the pathological significance of RCC.

\begin{tabular}{|c|c|c|c|c|c|}
\hline Pathological parameter & $\begin{array}{l}\text { Patient no. } \\
\qquad(\mathrm{n}=80)\end{array}$ & $\begin{array}{c}\text { GLUT2 } \\
\text { (range/p-value) }\end{array}$ & $\begin{array}{c}\text { GLUT3 } \\
\text { (range/p-value) }\end{array}$ & $\begin{array}{c}\text { GLUT4 } \\
\text { (range/p-value) }\end{array}$ & $\begin{array}{c}\text { GLUT5 } \\
\text { (range/p-value) }\end{array}$ \\
\hline \multicolumn{6}{|l|}{$\begin{array}{l}\text { Differentiation degree } \\
\text { (Furhman grade) }\end{array}$} \\
\hline Well & 15 & & & & \\
\hline Moderate & 45 & & & & $44.81 / 0.024$ \\
\hline Poor & 16 & & & $41.94 / 0.366$ & \\
\hline Indifferentiate & 4 & $52.88 / 0.384$ & $44.75 / 0.913$ & & \\
\hline \multicolumn{6}{|l|}{ Pelvic invasion } \\
\hline Yes & 9 & $48.89 / 0.329$ & & $50.28 / 0.240$ & $54.56 / 0.039$ \\
\hline No & 66 & & & & \\
\hline Undetermined & 5 & & $49.60 / 0.644$ & & \\
\hline \multicolumn{6}{|l|}{ Capsule breakage } \\
\hline Yes & 12 & $45.38 / 0.576$ & & $52.00 / 0.080$ & $54.75 / 0.019$ \\
\hline No & 64 & & & & \\
\hline Undetermined & 4 & & $49.60 / 0.637$ & & \\
\hline \multicolumn{6}{|l|}{ Tumor depth } \\
\hline 1 & 63 & & & & \\
\hline 2 & 4 & & & & $56.63 / 0.437$ \\
\hline 3 & 12 & $48.17 / 0.429$ & & & \\
\hline 4 & 1 & & $63.00 / 0.595$ & $77.00 / 0.029$ & \\
\hline \multicolumn{6}{|l|}{ Histological type } \\
\hline Clear cell & 57 & & $43.32 / 0.287$ & $41.01 / 0.990$ & $46.98 / 0.001$ \\
\hline Papillary & 6 & $50.83 / 0.416$ & & & \\
\hline Chromophobe & 15 & & & & \\
\hline Undetermined & 2 & & & & \\
\hline \multicolumn{6}{|l|}{ Tumor localization } \\
\hline Right & 40 & & & & \\
\hline Left & 39 & $41.23 / 0.551$ & $42.69 / 0.294$ & $41.01 / 0.669$ & $40.13 / 0.959$ \\
\hline Undetermined & 1 & & & & \\
\hline \multicolumn{6}{|l|}{ Vein invasion } \\
\hline Yes & 9 & $45.50 / 0.783$ & $63.00 / 0.321$ & & $63.00 / 0.312$ \\
\hline No & 67 & & & $40.63 / 0.615$ & \\
\hline Undetermined & 4 & & & & \\
\hline
\end{tabular}

filtration. Differences in fructose concentration between filtrate and intracellular compartment determines the direction of fructose flux mediated by GLUT5. Kidney GLUT5 protein levels are similar to those determined for GLUT5 in the intestine (19). Moreover, during the pre-natal period, levels of renal GLUT5 protein are low, but rapidly increase during weaning, when its expression is also inducible by the fructose diet (20).

GLUT5 is found in many tissues and its expression and activity are clearly regulated under malignant and nonmalignant pathological conditions. In the adult kidney, the regulation of GLUT5 expression is dependent and activity under physiological condition is fructose diet and pathological conditions such as hypertension and type 1 diabetes.

GLUT5 protein expression is affected by the development of tumors in certain organ systems. In general, oncogene-transformed cells that exhibit a cancerous pattern also experience an increase in hexose transport by overexpressing specific sugar transporters. In the present study, GLUT5 was overex- pressed in the majority of RCCs. This finding is not supported by previous studies, which showed GLUT1 to have the highest expression in RCCs (21). GLUT5 overexpression in RCCs could indicate other pathways for hexose metabolism in many RCCs.

GLUT5 was found overexpressed in a large-scale screening of the GLUT transporters: brain, breast, colon, liver, lung, testis and uterus tumors (22).

A marked increase in GLUT5 expression may indicate preferential utilization of fructose by RCC. This is associated with the need of tumors for an additional energy source.

Cancer cells maintain a high rate of glycolysis even in the presence of oxygen (Warburg effect) (23), probably due to the use of fermentative metabolism under the hypoxic conditions that develop in more aggressive tumors. GLUT5 overexpression may give rise to an increase in fructose uptake. Conversely, the increase in fructose usage by tumor cells may lead to GLUT5 overexpression in a positive feedback loop. 
Our findings support the importance of GLUT5 and the fructose metabolism in RCC. However, further studies must be conducted to elucidate the mechanism involved in sugar metabolism in RCC.

\section{Acknowledgements}

MBC has a grant from Fondo de Investigación Sanitaria (FIS), Instituto de Salud 'Carlos III'. Research in our Hospital is supported by the Fundación del Complejo Hospitalario Universitario A Coruña (CHUAC), Spain.

\section{References}

1. Mathew A, Devesa SS, Fraumeni JF Jr and Chow WH: Global increases in kidney cancer incidence, 1973-1992. Eur J Cancer Prev 11: 171-178, 2002.

2. Gupta K, Miller JD, Li JZ, Russell MW and Charbonneau C: Epidemiologic and socioeconomic burden of metastatic renal cell carcinoma (mRCC): a literature review. Cancer Treat Rev 34: 193-205, 2008

3. Ferlay J, Autier P, Boniol M, Heanue M, Colombet M and Boyle P: Estimates of the cancer incidence and mortality in Europe in 2006. Ann Oncol 18: 581-592, 2007.

4. American Cancer Society. Estimated new cancer cases and deaths by sex, US, 2008. American Cancer Society: http://www.cancer. org/downloads/stt/CFF2008Table_pg4.pdf. Accessed June, 2009.

5. Isselbacher KJ: Sugar and amino acid transport by cells in culture - differences between normal and malignant cells. N Engl J Med 286: 929-933, 1972.

6. Hatanaka M: Transport of sugars in tumor cell membranes. Biochim Biophys Acta 355: 77-104, 1974.

7. Kresnik E, Gallowitsch HJ, Mikosch P, et al: Fluorine-18fluorodeoxyglucose positron emission tomography in the preoperative assessment of thyroid nodules in an endemic goiter area. Surgery 133: 294-299, 2003.

8. Manolescu AR, Witkowska K, Kinnaird A, Cessford T and Cheeseman C: Facilitated hexose transporters: new perspectives on form and function. Physiology 22: 234-240, 2007.

9. Mueckler MA and Rhoads DB: Facilitative glucose transporters. Eur J Biochem 219: 713-725, 1994.

10. Schürmann A: Insight into the 'odd' hexose transporters GLUT3, GLUT5 and GLUT7. Am J Physiol Endocrinol Metab 295: 225-226, 2008.
11. Douard V and Ferraris RP: Regulation of the fructose transporter GLUT5 in health and disease. Am J Physiol Endocrinol Metab 295: 227-237, 2008.

12. Cantuaria G, Fagotti A, Ferradina G, Magalhaes A, Nadji M, Angioli R, Penalver M, Mancuso S and Scambia G: GLUT-1 expression in ovarian carcinoma: association with surival and response to chemotherapy. Cancer 92: 1144-1150, 2001.

13. Rogers ES, Macheda ML, Docherty SE, Carty MD, Henderson MA, Soeller WC, Gibbs EM, James DE and Best JD: Identification of a novel glucose transporter-like proteinGLUT-12. Am J Physiol Endocrinol Metab 282: 733-738, 2002.

14. Younes M, Lechago LV, Somoano JR, Mosharaf $M$ and Lechago J: Immunohistochemical detection of Glut3 in human tumors and normal tissues. Anticancer Res 17: 2747-2750, 1997.

15. Suganuma N, Segade F, Matsuzu K and Bowden DW: Differential expression of facilitative glucose transporters in normal and tumour kidney tissues. BJU Int 99: 1143-1149, 2007.

16. Lidgren A, Bergh A, Grankvist K, Rasmuson T and Ljungberg B: Glucose transporter-1 expression in renal cell carcinoma and its correlation with hypoxia inducible factor-1 $\alpha$. BJU Int 101: 480-484, 2007.

17. Miyakita H, Tokunaga M, Onda H, Usui Y, Kinoshita H, Kawamura N and Yasuda S: Significance of 18F-fluorodeoxyglucose positron emission tomography (FDG-PET) for detection of renal cell carcinoma and immunohistochemical glucose transporter 1 (GLUT-1) expression in the cancer. Int J Urol 9: 15-18, 2002.

18. Sugawara-Yokoo M, Suzuki T, Matsuzaki T, Naruse T and Takata K: Presence of fructose transporter GLUT5 in the S3 proximal tubules in the rat kidney. Kidney Int 56: 1022-1028, 1999.

19. Corpe CP, Bovelander FJ, Munoz CM, Hoekstra JH, Simpson IA, Kwon O, Levine M and Burant CF: Cloning and functional characterization of the mouse fructose transporter, GLUT5. Biochim Biophys Acta 1576: 191-197, 2002.

20. Burant CF and Saxena M: Rapid reversible substrate regulation of fructose transporter expression in rat small intestine and kidney. Am J Physiol Gastrointest Liver Physiol 267: G71, 1994.

21. Nagase Y, Takata K, Moriyama N, Aso Y, Murakami T and Hirano H: Immunohistochemical localization of glucose transporters in human renal cell carcinoma. Br J Urol 153: 798-801, 1995.

22. Godoy A, Ulloa V, Rodriguez F, et al: Differential subcellular distribution of glucose transporters GUT1-6 and GLUT9 in human cancer: ultrastuctural localization of GLUT1 and GLUT5 inbreast tumor tissues. J Cell Physiol 270: 614-627, 2006.

23. Warburg O: On the origin of cancer cells. Science 123: 309-314, 1956. 